Will a matchmaker invite her potential rival in?

Rupayan Pal and Vinay Ramani

Indira Gandhi Institute of Development Research, Mumbai August 2015

http://www.igidr.ac.in/pdf/publication/WP-2015-025.pdf 


\title{
Will a matchmaker invite her potential rival in?
}

\author{
Rupayan Pal and Vinay Ramani \\ Indira Gandhi Institute of Development Research (IGIDR) \\ General Arun Kumar Vaidya Marg \\ Goregaon (E), Mumbai- 400065, INDIA \\ Email(corresponding author): rupayan@igidr.ac.in
}

\begin{abstract}
This paper analyzes optimal strategies of an incumbent intermediary, who matches agents on the two sides of a market, in the presence of entry threat under alternative scenarios. It shows that, when entry is free, strategic entry accommodation is the optimal choice of the incumbent - not entry deterrence, unless the variation in agents' types is small. Entry accommodation remains optimal for the incumbent for a wide range of parametric configurations even when there is a fixed cost of entry. These results are in sharp contrast to the predictions of existing models of entry.
\end{abstract}

Keywords: Entry accommodation, Entry deterrence, Intermediation, Matching, Two-sided market

JEL Code: L12, L13, D43 


\title{
Will a matchmaker invite her potential rival in?
}

\author{
Rupayan Pal* Vinay Ramani ${ }^{\dagger}$
}

August 28, 2015

\begin{abstract}
This paper analyzes optimal strategies of an incumbent intermediary, who matches agents on the two sides of a market, in the presence of entry threat under alternative scenarios. It shows that, when entry is free, strategic entry accommodation is the optimal choice of the incumbent - not entry deterrence, unless the variation in agents' types is small. Entry accommodation remains optimal for the incumbent for a wide range of parametric configurations even when there is a fixed cost of entry. These results are in sharp contrast to the predictions of existing models of entry.

Keywords: Entry accommodation, Entry deterrence, Intermediation, Matching, Two-sided market

JEL Codes: L12, L13, D43
\end{abstract}

*Corresponding Author: rupayan@igidr.ac.in Address: Indira Gandhi Institute of Development Research (IGIDR), Film City Road, Santosh Nagar, Goregaon(E), Mumbai, Maharashtra, India 400065. Phone: 91-022-28416545, Fax: 91-022-28402752.

†vinay.ramani@iimu.ac.in. Address: Indian Institute of Management Udaipur (IIM-U), Polymer Science Building, MLSU Campus, Udaipur, Rajasthan, India 313001. Phone: 91-0294-2470100, Fax: 91-02942470126. 


\section{Introduction}

There are many markets where individuals on one side of the market use the services of intermediaries to get in contact with members on the other side of the market. Examples of such markets include the online dating market and the housing market. The online dating market is particularly interesting as the number of users of online dating services has been increasing year after year. A survey conducted by Pew Research Center and Statista.com found that annual revenue of the US online dating industry has grown from $\$ 43$ million in 2009 to nearly $\$ 295$ million in 2014 , and is projected to increase further to $\$ 400$ million by $2017 .{ }^{1}$ There are nearly 4000 firms in the online dating market. While firms like Match.com and eHarmony cater to the general audience and have a significant market share, there have also been a proliferation of niche dating sites. The online dating market in the UK is worth about 300 million pounds as of 2014 and there are nearly 1400 dating sites in UK alone. ${ }^{2}$ Increasing popularity of matrimonial websites, which assist in brokering marriages, in countries like India testifies that such matching market is growing in developing countries as well (Agrawal, 2015; Titzamann, 2013; Shako, 2004). ${ }^{3}$ Interestingly, unlike most online businesses, an average matrimonial website collects about 95 percent of its revenues by charging user fees, while remaining five percent of its revenues come from advertisements. ${ }^{4}$ These online matching intermediaries basically lower the search cost for users of these services and create marketplaces where an individual can meet other

\footnotetext{
${ }^{1}$ Source: http://www.pewresearch.org/fact-tank/2015/04/20/5-facts-about-online-dating/ and http://www.statista.com/statistics/245333/us-mobile-dating-market-revenue/.

${ }^{2}$ Source: UK Online Dating Association (ODA).

${ }^{3}$ India's online matchmaking industry, which took off in the late 1990s, became worth of about INR 4 billion in 2011 and is estimated to grow further by 27 percent per year. Source: Online Matrimony Market in India 2012, Netscribes (India) Pvt. Ltd. , published in September 2012, http://marketpublishers.com/report/technologies_electronics/telecommunications/online-matrimonymarket-in-india-2012.html.

${ }^{4}$ Source: "Booming biz of online marriages", Business Standard, December 22, 2011. http://www.business-standard.com/article/companies/booming-biz-of-online-marriages111122200022_1.html .
} 
people. Furthermore, the presence of multiple intermediary firms in this market implies that incumbent firms are able to accommodate the entry of new firms. It is this issue of entry accommodation that is of interest to us in this paper.

We consider competition in a model of intermediation, where an incumbent matchmaker faces the threat of entry from a potential entrant. The models of intermediation gained prominence after the seminal work of Gale and Shapley (1968), and Roth and Sotomayor (1990). Caillaud and Julien (2001a, 2003) study a model of competition between cybermediaries, where competition is characterized by asymmetric network externalities. In our model we do not focus on the issue of network externalities, instead we model the issue of entry. More recent work on intermediation include Armstrong (2006) and Lee (2013, 2014). In this paper we ask the following question - Is entry deterrence an optimal strategy for the incumbent? If there is no fixed cost of entry, then entry deterrence requires that the incumbent firm intermediate the entire market. However, there is an alternative strategy for the incumbent that may be more profitable. By strategically accommodating the entrant, the incumbent can earn a higher profit. This occurs when the variation in agents', i.e., match seekers', types is greater than a critical level. Now consider the case where the entrant has a fixed cost of entry. Intuitively, if the incumbent finds it profitable to deter entry when there is free entry, then it should be able to deter entry more easily when there is fixed cost of entry. We show that there is a wide range of parameter configurations such that strategic entry accommodation remains optimal for the incumbent even when there is fixed cost of entry.

It is well documented in the literature that, in one-sided markets, entry of new firms results in lower output of the incumbent firm as well as lower price. As a result, entry deterrence is generally preferred by the incumbent firm than entry accommodation and, thus, the incumbent firm often adopts anti-competitive strategies in order to deter entry. In their early studies, Spence (1977, 1979), Dixit (1979, 1980), Fudenberg and Tirole (1984) examine the efficacy of excess capacity, over production and over investment as entry deterring strategies and discuss the taxonomy of blockaded entry, deterred entry, 
and accommodated entry. Milgrom and Roberts (1981) argue that the incumbent firm can engage in limit pricing in order to deter entry under asymmetric information regarding the incumbent's marginal cost of production. The incumbent firm may also sign contracts with the buyers and/or input suppliers in order to deter entry (Aghion and Bolton, 1987; Dewatripont, 1987 \& 1988; Pal and Saha, 2006 \& 2008). In contrast, we show that in a two-sided market the incumbent can strategically engage in a contract with a subset of heterogeneous buyers, which enables the incumbent to charge higher price, and obtain higher profit by inducing entry of new firm in the market compared to that under entry deterrence.

Closest to the set-up of our model is Ramani (2015). In Ramani (2015) the main objective is to justify a stylized fact of the online dating market - that there is market segmentation present. That is, in equilibrium, there are multiple intermediaries present, each of whom caters to different segments of the market. This finding is also confirmed in a study by Ahuvia and Adelman (1992) who state that "As the market for introduction services has become more crowded, these services have increasingly targeted narrower market niches such as ethnic groups, people with specific interests, the very rich, or marginalized groups such as the disabled". The discussion in Ramani (2015) is on the issue of what happens to the segments and the optimal pricing strategies of firms when entry is accommodated, and when entry is deterred. It shows that if entry is accommodated, then segmentation naturally arises, and if entry is deterred, then the incumbent chooses to intermediate a section of the market such that doing so leaves the entrant with zero profit. But then, this raises a fundamental question - would the incumbent prefer to accommodate entry or would it prefer to deter entry? We specifically address this question here.

The remainder of the paper is organized as follows. Section 2 states the model and introduces relevant notation. Section 3 discusses the issue of strategic entry accommodation and entry deterrence. Section 4 concludes. For ease of exposition, all proofs have been reported in the Appendix. 


\section{The Model}

Consider a matching market where an incumbent intermediary $I$ faces the threat of entry from a potential entrant $E$. The entrant incurs a fixed cost of entry $K$. We analyze two cases: (i) $K=0$ and (ii) $K>0$. The intermediaries create marketplaces where agents from two sides of the market (say men and women) can meet. The agents differ in certain verifiable characteristics (say age, education, income, health, etc) and can be represented by a one dimensional characteristic called type. We assume that the types are distributed uniformly on $[a, b] \subset[0, \infty)$ and higher type agents are considered to be better. Further, in line with the literature on positive assortative matching (see Becker, 1973), we consider complementarity in match value; i.e. a match between a type $x$ agent and a type $y$ agent generates a match value of $\mu(x, y)=x y$ to both of the types. This implies that each agent strictly prefers to be matched with a higher type agent. Intermediaries do not observe the types of the agents. Therefore an intermediary cannot price discriminate and charges the same price to the interval of agents that it decides to match. The ex ante expected utility of a type $x$ agent from accepting a contract from intermediary $i$ is $x E\left[y \mid y \in[r, s]_{x}\right]-p_{i}$, where $[r, s]_{x}$ denotes the interval of potential mates assigned to type $x$, and $p_{i}$ is the price charged by the intermediary $i, i=I, E$.

The incumbent moves first and offers a contract to a subset of men and women. The entrant observes the actions of the incumbent and then chooses to "enter" or "stay out". If there is entry, the entrant then offers a contract to a subset of men and women. After contracts have been offered by the firms, the agents decide whether to accept or reject their contract. Finally, intermediaries match the agents.

The basic structure of our game is similar to Ramani (2015), where it is shown that there exists optimal thresholds $c_{1}, c_{2} \in[a, b]$, such that all types in the interval $\left[c_{2}, b\right]$ are offered a contract only by the incumbent, and all types in the interval $\left[c_{1}, c_{2}\right)$ are offered a contract only by the entrant. This equilibrium is supported by the following belief system that the residual set of types who state their preferences to the entrant consists only of those types 
who do not receive a contract from the incumbent. ${ }^{5}$

\section{Entry Deterrence and Entry Accommodation}

Our interest in this paper is in studying the strategic aspects of entry deterrence and entry accommodation. Is accommodation in this market ever an optimal strategy for the incumbent? We answer this question by considering two alternative scenarios, $K=0$ and $K>0$, as mentioned before. When $K=0$, entry deterrence requires the incumbent to match all the types. However, when $K>0$, the incumbent can strategically choose to intermediate in an interval $\left[\hat{c}_{2}^{*}, b\right]$, where $\hat{c}_{2}^{*}>a$, and still deter entry. Intuitively, if the incumbent finds it profitable to deter the entrant when $K=0$ then when $K>0$ it can improve its profit by choosing $\hat{c}_{2}^{*}>a$ so that the residual types $\left[a, c_{2}^{*}\right)$ left for the entrant do not provide the entrant with a profit larger than $K$.

Before proceeding with the analysis, we first state the optimization problem of the incumbent and the entrant. The incumbent moves first and optimally chooses a cut-off type $c_{2}$, and the entrant chooses the cut-off type $c_{1}$. Given a $c_{2}>a$ being chosen by the incumbent, the entrant's problem is as follows.

$$
\begin{aligned}
\operatorname{Max}_{c_{1}} & \pi_{E}^{a c c}\left(c_{1}, c_{2}\right)=\left[F\left(c_{2}\right)-F\left(c_{1}\right)\right] p_{E}-K \\
& \text { subject to the constraints } \\
& a \leq c_{1}<c_{2}<b \\
& \int_{c_{1}}^{c_{2}} \frac{c_{1} z f(z) d z}{F\left(c_{2}\right)-F\left(c_{1}\right)}-p_{E} \geq 0
\end{aligned}
$$

Basically, the entrant has to ensure that the lowest type in the interval $\left[c_{1}, c_{2}\right)$ participates. Let us denote the solution of problem (1) by $c_{1}=c_{1}\left(c_{2}\right)$. As the match value exhibits

\footnotetext{
${ }^{5}$ Note that in our model, multiple equilibria is possible depending on the particular belief system. In particular, if there are beliefs that all contracts will be rejected, then obviously there is no entry. However, such a case is uninteresting.
} 
complementarity, if the lowest type participates, all types $x>c_{1}$ also participate. The incumbent's problem is as follows.

$$
\begin{aligned}
\operatorname{Max}_{c_{2}} \pi_{I}^{a c c}\left(c_{1}, c_{2}\right) & =\left[1-F\left(c_{2}\right)\right] p_{I}, \\
\text { subject to the constraints } & \\
a & <c_{2}<b \\
\int_{c_{2}}^{b} \frac{c_{2} z f(z) d z}{1-F\left(c_{2}\right)}-p_{I} & \geq \int_{c_{1}}^{c_{2}} \frac{c_{1} z f(z) d z}{F\left(c_{2}\right)-F\left(c_{1}\right)}-p_{E} \\
c_{1} & =c_{1}\left(c_{2}\right)
\end{aligned}
$$

where the incumbent also incorporates the incentive compatibility constraint for type $c_{2}$, and the entrant's optimal choice of $c_{1}$ for any given $c_{2}$.

Since the participation constraint for the entrant's problem and the incentive compatibility constraint for the incumbent's problem have to bind, prices charged by the incumbent $\left(p_{I}\right)$ and the entrant $\left(p_{E}\right)$, for any given $c_{1}$ and $c_{2}\left(a \leq c_{1}<c_{2}<b\right)$, in the case of entry accommodation are $p_{I}=\frac{b c_{2}+c_{1}^{2}}{2}$ and $p_{E}=\frac{c_{1}\left(c_{2}+c_{1}\right)}{2}$, respectively. Clearly, an intermediary can charge a higher price, if it serves a relatively higher segment of the market: $\frac{\partial p_{I}}{\partial c_{2}}>0$, $\frac{\partial p_{E}}{\partial c_{1}}>0$ and $\frac{\partial p_{E}}{\partial c_{2}}>0$. It also follows that we can rewrite the problem of the entrant and the incumbent as:

$$
\begin{aligned}
\underset{c_{1}}{\operatorname{Max}} & \pi_{E}^{a c c}\left(c_{1}, c_{2}\right)=\int_{c_{1}}^{c_{2}} c_{1} z f(z) d z=\frac{c_{1} c_{2}^{2}-c_{1}^{3}}{2(b-a)}, \\
& \text { subject to the constraints } \\
& a \leq c_{1}<c_{2}<b .
\end{aligned}
$$

and

$$
\begin{aligned}
& \underset{c_{2}}{\operatorname{Max}} \pi_{I}^{a c c}\left(c_{1}, c_{2}\right)=\int_{c_{2}}^{b} c_{2} z f(z) d z-\int_{c_{1}}^{c_{2}} \frac{\left(c_{2}-c_{1}\right)\left[1-F\left(c_{2}\right)\right]}{F\left(c_{2}\right)-F\left(c_{1}\right)} z f(z) d z=\frac{\left(b-c_{2}\right)\left(b c_{2}+c_{1}^{2}\right)}{2(b-a)} \\
& \text { subject to the constraints } \\
& a<c_{2}<b \\
& c_{1}=c_{1}\left(c_{2}\right)
\end{aligned}
$$




\subsection{Case 1: $K=0$}

Proposition 1. The necessary and sufficient condition for the incumbent to deter entry is that it chooses $p_{I}^{*}=\int_{a}^{b} a z f(z) d z$ and intermediate the entire market; i.e. $c_{2}^{*}=a$.

Proof: Please see the Appendix.

From Proposition 1 we know that by serving the entire market, i.e., by choosing $c_{2}=a$, the incumbent can deter entry and earn profit

$$
\pi_{I}^{\text {det }}=[1-F(a)] p_{I}=\int_{a}^{b} a z f(z) d z=\frac{1}{2} a(a+b) .
$$

On the other hand, the incumbent can induce entry by not serving the entire market, i.e., by choosing $c_{2}>a$. The question is, is it always optimal for the incumbent to deter entry? In other words, does entry deterrence necessarily result in higher profit of the incumbent than that from entry accommodation?

Note that, in the case of entry accommodation, the problem of the entrant in the post entry scenario can be written as in (3). Solving the above problem, we get

$$
c_{1}=\left\{\begin{array}{l}
\frac{c_{2}}{\sqrt{3}}, \text { if } a<\frac{c_{2}}{\sqrt{3}}<b \\
a, \text { if } \frac{c_{2}}{\sqrt{3}} \leq a
\end{array} .\right.
$$

Therefore, we can rewrite the incumbent's problem as follows.

$$
\begin{aligned}
\underset{c_{2}}{\operatorname{Max}} & \pi_{I}^{a c c}\left(c_{1}, c_{2}\right)=\frac{\left(b-c_{2}\right)\left(b c_{2}+c_{1}^{2}\right)}{2(b-a)}, \\
& \text { subject to the constraints } \\
& a<c_{2}<b \text { and }(2) .
\end{aligned}
$$


Solving problem (7) we get the following (please see the Appendix for details).

$$
\begin{aligned}
& c_{2}=\frac{1}{3}(\sqrt{13}-2) b \text { and } c_{1}=\frac{1}{3 \sqrt{3}}(\sqrt{13}-2) b, \text { if } 3.23637 a<b \\
& c_{2}=\frac{b^{2}-a^{2}}{2 b} \text { and } c_{1}=a, \text { if } 2.4142 a<b \leq 3.23637 a \\
& c_{2}=a+\epsilon \text { and } c_{1}=a, \text { if } a<b \leq 2.4142 a ; \text { where } \epsilon \text { is a small positive number. }
\end{aligned}
$$

Clearly, the equilibrium choices of $c_{1}$ and $c_{2}$ are crucially dependent on relative magnitudes of $a$ and $b$, i.e., on the extent of variation in agents' types. Substituting values of the equilibrium $c_{1}$ and $c_{2}$, we get the incumbent's profit under entry accommodation as follows.

$$
\pi_{I}^{a c c}=\left\{\begin{array}{l}
\frac{(13 \sqrt{13}-35) b^{3}}{81(b-a)}, \quad \text { if } 3.23637 a<b \\
\frac{\left(a^{2}+b^{2}\right)^{2}}{8 b(b-a)}, \quad \text { if } 2.4142 a<b \leq 3.23637 a \\
\frac{(b-a-\epsilon)[a(a+b)+b \epsilon]}{2(b-a)}, \quad \text { if } a<b \leq 2.4142 a .
\end{array}\right.
$$

From (5) and (8) the following result is immediate.

Proposition 2. Consider the entry game between the incumbent and the entrant, where the type distribution is Uniform on $[a, b]$. If $b \geq 2.414 a$, entry is accommodated, and if $b<2.414 a$ entry is deterred.

Proof: Please see the appendix.

It is evident that, if $a=0$, then for any $b>0$ entry accommodation is the optimal strategy for the incumbent. However, if $a>0$, then whether the incumbent will accommodate entry or deter entry that depends on the relative magnitude of $b$. If $b$ is sufficiently greater than $a$, then the incumbent is better off by accommodating the entrant, while it is better off deterring entry otherwise. For the example, where the type distribution is uniform on $[1,2]$, $\pi_{I}^{a c c}=\frac{3-\epsilon-2 \epsilon^{2}}{2}<\pi_{I}^{d e t}=\frac{3}{2}$, since $\epsilon>0$. However, where the type distribution is uniform on $[1,10], \pi_{I}^{a c c}=16 \cdot 3>\pi_{I}^{d e t}=5 \cdot 5$. That is, in equilibrium, whether the incumbent will deter 
entry or accommodate the entrant that depends not only on the degree of complementarity between the types but also on the set of types $[a, b]$ in the market.

While we have considered the case of a uniform distribution in this paper, the proof is similar for a distribution for which the density function is increasing or a distribution for which the density function is decreasing, though the critical $b$ is different in each case. If the density of types is increasing on $[a, b]$ then the market has a greater concentration of high types and the incumbent will accommodate the entrant for a critical $b$ smaller than 2.4142a. This is because, with greater concentration of high types, deterring entry is more costly as it reduces the fee that the incumbent can charge, whereas by accommodating the entrant the incumbent can charge a larger fee to the higher concentration of high types in the market. If the density function is decreasing on $[a, b]$ then the critical value of $b$ above which entry is accommodated is greater than that for a uniform distribution, because there is a greater concentration of lower types.

Proposition 2 presents an important result of this paper; viz. the incumbent can earn a higher profit by strategically accommodating the entrant, unless the coefficient of variation of types of agents in the market is less than a critical level. ${ }^{6}$ Entry accommodation is strategic because the incumbent decides the subset of types to whom the entrant can offer a contract, although it is feasible for the incumbent to serve the entire market and deter entry. Given the belief system of the game, in the case of entry accommodation, the incumbent's choice of $c_{2}^{*}$ leaves a residual population of types $\left[a, c_{2}^{*}\right)$ who state their preferences to the entrant. The entrant then chooses a cut off threshold $c_{1}^{*}$ optimally and offers a contract to the subset of types in $\left[c_{1}^{*}, c_{2}^{*}\right)$ and earns strictly positive profit.

Interestingly, while entry deterrence causes the incumbent to be a monopolist, it still cannot charge the monopoly fee that it could charge in absence of entry threat. In a sense this is similar to the argument in the contestable markets literature wherein a firm in spite of being single in the market cannot charge the monopoly price. It is argued that the mere

\footnotetext{
${ }^{6} b<2.4142 a \Rightarrow$ (Coefficient of variation $)<\frac{2.4142-1}{(2.4142+1) \sqrt{3}}=0.239145$.
} 
"threat of entry" affects the behavior of the incumbent firm and that "in the absence of actual competition, potential competition is very effective in disciplining the incumbent firms" (Baumol et al, 1982). In the present context, if there is no threat of entry, then the monopolist does not offer contracts to all the types in $[a, b]$, instead it chooses an interior marginal type $c^{m}>a$, which is similar to that in the case of contestable markets. The reason is, the higher is the marginal type, the greater is the fee that the incumbent can charge. However, unlike the case of contestable markets, the presence of the entrant leaves the incumbent with two options: it can either deter entry and be a monopolist only by ensuring the participation of the lowest type $a$, or share the market with the entrant. If the cost of entry deterrence (in terms of lost profits) is large enough, then the incumbent prefers to accommodate the entrant. This result is in sharp contrast to the findings of existing studies on entry threats in oligopoly.

\subsection{Case 2: $K>0$}

We now turn to analyze the scenario in which there is a fixed cost $K(>0)$ of entry. Note that, if the entrant enjoys absolute monopoly power in the post entry scenario, the problem of the entrant can be written as $\underset{c_{1}}{\operatorname{Max}} \pi_{E}^{a c c}\left(c_{1}, b\right)$, subject to the constraint $a \leq c_{1}<b$; where $\pi_{E}^{a c c}\left(c_{1}, b\right)=\int_{c_{1}}^{b} c_{1} z f(z) d z-K=\frac{c_{1}\left(b^{2}-c_{1}^{2}\right)}{2(b-a)}-K$. Solving this problem, we get $c_{1}^{* M}=$ $\left\{\begin{array}{l}\frac{b}{\sqrt{3}}, \text { if } b>\sqrt{3} a \\ a, \text { if } b<\sqrt{3} a\end{array}\right.$ and $\pi_{E}^{a c c}\left(c_{1}^{* M}, b\right) \leq 0 \Leftrightarrow K \geq \hat{K}=\left\{\begin{array}{l}\frac{b^{3}}{3 \sqrt{3}(b-a)}, \text { if } b>\sqrt{3} a \\ \frac{a(a+b)}{2}, \text { if } b<\sqrt{3} a\end{array}\right.$. That is, if the fixed cost of entry is sufficiently large $(K \geq \hat{K})$, entry is blockaded. In other words, the possibility of entry arises only when $K<\hat{K}$.

Since it is always feasible for the incumbent to deter entry in the case of no entry fee $(K=0)$, it is evident that the incumbent can do so more comfortably when there fixed entry cost $(K>0)$. That is, unlike as in the case of $K=0$, the incumbent need not necessarily serve the entire market in order to deter entry. 
Proposition 3. (a) When $K \geq \hat{K}$, entry is blockaded. (b) When $0<K<\hat{K}$, there exists $a \hat{c}_{2} \in(a, b)$ such that (i) any $c_{2} \in\left[a, \hat{c}_{2}\right]$ deters entry and (ii) if $c_{2} \in\left(\hat{c}_{2}, b\right]$, entry occurs.

Proof: Please see the Appendix.

Proposition 3 states that the incumbent can always deter entry by choosing $c_{2}$ appropriately. Note that for any given fixed cost $K$, if entry occurs, the entrant's problem in the Stage 2 of the game is as follows.

$$
\underset{c_{1}}{\operatorname{Max}} \pi_{E}\left(c_{1}, c_{2}\right)=\int_{c_{1}}^{c_{2}} c_{1} z f(z) d z-K=\frac{c_{1} c_{2}^{2}-c_{1}^{3}}{2(b-a)}-K
$$

subject to the constraints

$$
a \leq c_{1}<c_{2}<b
$$

Clearly, the solution of the above problem is the same as that in (3). It implies that, in the event of entry, the entrant's choice of $c_{1}$ can be written as follows.

$$
\tilde{c}_{1}=\left\{\begin{array}{l}
a, \text { if } 0<a \leq c_{2}<b \leq \sqrt{3} a \text { or } 0<a \leq c_{2} \leq \sqrt{3} a<b \\
\frac{c_{2}}{\sqrt{3}}, \text { if } 0<\sqrt{3} a<c_{2}<b .
\end{array}\right.
$$

Corresponding profit of the entrant is $\tilde{\pi}_{E}=\left\{\begin{array}{l}\frac{a\left(c_{2}^{2}-a^{2}\right)}{2(b-a)}-K, \text { if } \tilde{c}_{1}=a \\ \frac{c_{2}^{3}}{3 \sqrt{3}(b-a)}-K, \text { if } \tilde{c}_{1}=\frac{c_{2}}{\sqrt{3}}\end{array}\right.$. Therefore, in order to deter entry the incumbent needs to choose $c_{2}$ such that $\tilde{\pi}_{E} \leq 0$. It is straight forward to observe that

$$
\tilde{\pi}_{E} \leq 0 \Rightarrow c_{2} \leq \tilde{c_{2}}=\left\{\begin{array}{l}
\frac{\sqrt{a^{3}+2 K(b-a)}}{\sqrt{a}}=c_{2}^{\prime}, \text { if } \tilde{c}_{1}=a \\
\sqrt{3}(b-a)^{\frac{1}{3}} K^{\frac{1}{3}}=c_{2}^{\prime \prime}, \text { if } \tilde{c}_{1}=\frac{c_{2}}{\sqrt{3}} .
\end{array}\right.
$$

Clearly, any $c_{2} \in\left[a, \tilde{c}_{2}\right]$ deters entry. But, in absence of entry threat, the incumbent's optimal choice of the marginal consumer is as follows.

$$
\left.\bar{c}_{2}=\underset{c_{2}}{\operatorname{ArgMax}}\left\{\pi_{I}^{\text {det }}=\int_{c_{2}}^{b} c_{2} z f(z) d z=\frac{c_{2}\left(b^{2}-c_{2}^{2}\right)}{2(b-a)}\right\}\right]=\left\{\begin{array}{l}
\frac{b}{\sqrt{3}}, \text { if } b>\sqrt{3} a \\
a, \text { if } b<\sqrt{3} a
\end{array}\right.
$$


Therefore, if the incumbent wants to deter entry, it will serve agents of type $c_{2}^{\text {det* }}$ and higher, where

$$
c_{2}^{\operatorname{det} *}=\operatorname{Max}\left\{\operatorname{Min}\left\{\bar{c}_{2}, \tilde{c}_{2}\right\}, a\right\} .
$$

Upon inspection, we obtain the following.

Corollary 1. In the case of entry deterrence, the incumbent serves only $\left[c_{2}^{\text {det* }}, b\right]$ segment of the market and obtain profit $\pi_{I}^{\text {det* }}$ in the equilibrium, where $c_{2}^{\text {det* }}$ and $\pi_{I}^{\text {det* }}$ are as follows.

(a) If $0<a<b<\sqrt{3} a, c_{2}^{\text {det* }}=a$ and $\pi_{I}^{\text {det* }}=\frac{a(a+b)}{2}$ for any $K \geq 0$.

(b) If $0<\sqrt{3} a<b \leq 3 a$ and $0<K<\frac{a\left(b^{2}-3 a^{2}\right)}{6(b-a)}, c_{2}^{\text {det* }}=c_{2}^{\prime}$ and $\pi_{I}^{\text {det* }}=\frac{\left(a^{2}+a b-2 K\right) \sqrt{a^{3}+2 K(b-a)}}{2 a^{3 / 2}}$.

(c) If $0<\sqrt{3} a<b \leq 3 a$ and $\frac{a\left(b^{2}-3 a^{2}\right)}{6(b-a)} \leq K<\hat{K}, c_{2}^{\text {det } *}=\frac{b}{\sqrt{3}}$ and $\pi_{I}^{\text {det* }}=\frac{b^{3}}{3 \sqrt{3}(b-a)}$.

(d) If $0<3 a<b$ and $0<K \leq \frac{a^{3}}{b-a}$, $c_{2}^{\text {det* }}=c_{2}^{\prime}$ and $\pi_{I}^{\text {det* }}=\frac{\left(a^{2}+a b-2 K\right) \sqrt{a^{3}+2 K(b-a)}}{2 a^{3 / 2}}$.

(e) If $0<3 a<b$ and $\frac{a^{3}}{(b-a)}<K<\frac{b^{3}}{27(b-a)}, c_{2}^{\text {det* }}=c_{2}^{\prime \prime}$ and $\pi_{I}^{\text {det* }}=\frac{\sqrt{3}}{2}\left\{\frac{b^{2} K^{1 / 3}}{(b-a)^{2 / 3}}-3 K\right\}$.

(f) If $0<3 a<b$ and $\frac{b^{3}}{27(b-a)} \leq K<\hat{K}, c_{2}^{\text {det* }}=\frac{b}{\sqrt{3}}$ and $\pi_{I}^{\text {det* }}=\frac{b^{3}}{3 \sqrt{3}(b-a)}$.

Proof: Please see the Appendix.

From Corollary 1 it is evident that, when $0<K<\hat{K}$, the incumbent would strategically choose $c_{2}^{\text {det* }}>a$ in order to deter entry, unless the variation in agents' type is too small $(b<\sqrt{3} a) \cdot{ }^{7}$ In the latter case, i.e. if $b<\sqrt{3} a$, entry can be deterred by choosing $c_{2}>a$, but the incumbent can obtain higher profit by setting $c_{2}=a$. Further, note that, when $0<\sqrt{3} a<b, c_{2}=\frac{b}{\sqrt{3}}$ maximizes monopoly profit of the incumbent in absence of entry threat and $c_{2}^{\text {det* }} \leq \frac{b}{\sqrt{3}}$. As a result, if $0<\sqrt{3} a<b$, entry deterring profit of the incumbent in the presence of fixed cost of entry is higher than that in Case 1. Otherwise, if $b<\sqrt{3} a$, by deterring entry the incumbent obtains the same level of profit as that in Case 1.

\footnotetext{
${ }^{7} b<\sqrt{3} a \Rightarrow$ (Coefficient of variation) $=0.154700538$.
} 
On the other hand, it is easy to observe that, if the incumbent wants to accommodate entry, it needs to leave out a larger segment of the market for the entrant when there is a fixed cost of entry compared to that in absence of the fixed cost. This is because, in order to accommodate entry the incumbent needs to choose $c_{2} \in\left(\tilde{c}_{2}, b\right)$ and $\frac{\partial \tilde{c}_{2}}{\partial K}>0$. In other words, entry accommodation calls for decrease in the incumbent's market share in the present scenario compared to that in Case 1, which has a negative effect on incumbent's profit. However, since the incumbent now serves relatively higher type agents only, it can charge higher fees than in Case 1, which has a positive effect on its profits. The net effect of entry accommodation on incumbent's profit depends on relative magnitudes of $a$ and $b$, i.e., on the extent of variation in agents' types, as well as the amount of fixed cost of entry. Formally, we can write the incumbent's problem, if it wants to accommodate entry when there is fixed cost of entry, as follows.

$$
\begin{aligned}
& \underset{c_{2}}{\operatorname{Max}} \pi_{I}^{a c c}\left(c_{1}, c_{2}\right)=\frac{\left(b-c_{2}\right)\left(b c_{2}+c_{1}^{2}\right)}{2(b-a)} \\
& \text { subject to the constraints } \\
& \text { (i) } a<c_{2}<b \\
& \text { (ii) } c_{1}=\tilde{c}_{1} \text { and } \\
& \text { (iii) } c_{2}>\tilde{c_{2}} \text {. }
\end{aligned}
$$

Solving the above problem and comparing the equilibrium profit of the incumbent under entry accommodation, $\pi_{I}^{a c c *}$, with that under entry deterrence, $\pi_{I}^{\text {det* }}$, we obtain the following.

Proposition 4. Consider the entry game between the incumbent and the entrant in which the type distribution is Uniform on $[a, b]$. Then, in the presence of fixed cost of entry, we have the following;

(a) It is optimal for the incumbent to accommodate entry, unless $b<2.414 a$ or the entrant's fixed cost $K>\bar{K} \in\left(0, \frac{b^{3}}{27(b-a)}\right)$,

(b) Entry is deterred in the equilibrium, regardless of relative magnitudes of a and $b$ (mag- 
nitude of the entrant's fixed cost), if $K \geq \frac{b^{3}}{27(b-a)}(b<2.414 a)$

Proof: Please see the Appendix.

Proposition 4 states that the incumbent prefers to accommodate entry over entry deterrence for a large number of parametric configurations, even when there is a fixed cost of entry. The greater the variation in agents' types and/or lower the entrant's fixed cost, the greater is the possibility of entry accommodation in the equilibrium.

\section{Conclusion}

This paper presents a framework for analyzing competition between intermediaries by considering a model of intermediation where two matchmakers, an incumbent and an entrant compete for market share by offering contracts to match types of agents on the two sides of the market. Contrary to the prediction of existing models of entry, this paper demonstrates that entry accommodation, not entry deterrence, is often the optimal strategy of the incumbent. It implies that coexistence of multiple intermediaries in a market need not necessarily be due to incumbent's inability to effectively deter entry, unlike as commonly perceived. This result highlights the importance of considering specific characteristics of the market while formulating business strategies and entry regulations.

In this paper we have assumed a particular belief system of agents', i.e., of buyers', which leads to market segmentation in the case of entry accommodation. It might be interesting to extend the analysis to allow for endogenous market segmentation without relying on agents' prior beliefs. It also seems to be interesting to examine the social welfare implications of entry accommodation and entry deterrence in the case of two-sided markets. We leave these for future research. 


\section{Appendix}

Proof of Proposition 1: We first prove sufficiency. If $c_{2}^{*}=a$ and $p_{I}^{*}=\int_{a}^{b} a z f(z) d z$, then type $a$ is indifferent between accepting the incumbent's contract and remaining single and so will accept the contract. The expected utility to any type $x>a$ from accepting the incumbent's contract is $\int_{a}^{b} x z f(z) d z-p_{I}^{*}=\int_{a}^{b}(x-a) z f(z) d z>0$. Therefore if type $a$ participates, then all types participate. Now the entrant faces a residual market which is empty. Therefore there is no entry.

To prove necessity, suppose now that $c_{2}^{*}>a$. The entrant then faces a residual market $\left[a, c_{2}^{*}\right)$. From Lemma 2, if $c_{2}^{*}$ is such that $\int_{a}^{c_{2}^{*}} z f(z) d z-a^{2} f(a)>0,{ }^{8}$ then there exists a $c_{1}^{*} \in\left(a, c_{2}^{*}\right)$ and $p_{E}^{*}$ such that the contract $\left(\left[c_{1}^{*}, c_{2}^{*}\right), p_{E}^{*}\right)$ earns a positive profit for the entrant. Hence there is entry.

Setting $p_{I}^{*}<\int_{a}^{b} a z f(z) d z$ is not an optimal strategy for the incumbent in any Nash equilibrium because the incumbent improves its profit by increasing $p_{I}^{*}$ to $\int_{a}^{b} a z f(z) d z$ and still ensures that type $a$ participates. If $p_{I}^{*}>\int_{a}^{b} a z f(z) d z$, then type $a$ does not participate as the expected utility of type $a$ is $\int_{a}^{b} a z f(z) d z-p_{I}^{*}<0$. Therefore, at an equilibrium if $p_{I}^{*}>\int_{a}^{b} a z f(z) d z$, then $c_{2}^{*}>a$. But then, by the earlier argument, $c_{2}^{*}>a$ causes the entrant to enter.

Proof of Proposition 2: Note that, for any given $c_{2}(>a)$, the entrant's optimal choice of $c_{1}$ in the post entry scenario is as in (3).

First, let us consider that $a \leq \frac{c_{2}}{\sqrt{3}}<b$. Then, the incumbent's problem in Stage 1 of the game can be written as $\underset{c_{2}}{\operatorname{Max}} \pi_{I}^{a c c}\left(c_{1}, c_{2}\right)$, subject to the constraints $c_{1}=\frac{c_{2}}{\sqrt{3}}$ and $\left.a<c_{2}<b\right]$. Solving this problem, we get $c_{2}=\left\{\begin{array}{l}\frac{1}{3}(\sqrt{13}-2) b=\hat{c_{2}} \text {, if } b>\frac{3 a}{\sqrt{13}-2}=1.86852 a \\ a, \text { if } b \leq \frac{3 a}{\sqrt{13}-2}\end{array}\right.$. Now,

${ }^{8}$ Note that if $c_{2}^{*}$ is such that $\int_{a}^{c_{2}^{*}} z f(z) d z-a^{2} f(a)<0$ for the entrant's problem, then the profitmaximizing value for $c_{1}$ is $c_{1}^{*}=a$. 
when $c_{2}=\hat{c_{2}}$, (i) $a \leq \frac{c_{2}}{\sqrt{3}}<b$, if $b>3.23637 a$, and (ii) $\pi_{I}^{a c c}-\pi_{I}^{d e t}=\frac{81 a^{3}-81 a b^{2}+(26 \sqrt{13}-70) b^{3}}{162(b-a)}$, which is positive if either $b>3.04292 a$ or $b<1.25892 a$. Clearly, when $b>3.23637 a$, we have $c_{2}=\hat{c_{2}}, c_{1}=\frac{\hat{c_{2}}}{\sqrt{3}}$, entry is accommodated and $\pi_{I}^{a c c}>\pi_{I}^{\text {det }}$.

When $1.86852 a<b<3.23637 a$, if $c_{2}=\hat{c_{2}}, c_{1}=a$. But, if $c_{1}=a$, the incumbent's problem can be written as $\underset{c_{2}}{\operatorname{Max}} \pi_{I}^{a c c}\left(c_{1}, c_{2}\right)$, subject to the constraints $c_{1}=a$ and $\left.a<c_{2}<b\right]$. Solving this problem, we get $c_{2}=\frac{b^{2}-a^{2}}{2 b}=c_{2}^{0}$, say. It is easy to check that (i) $a<c_{2}^{0}<b$, if $b>2.4142 a$, and (ii) $a<\frac{c_{2}^{0}}{\sqrt{3}}<b$, if $b>3.73205 a$. Therefore, when $2.4142 a<b<3.23637 a$, $c_{2}=c_{2}^{0}, c_{1}=a$, entry is accommodated and $\pi_{I}^{a c c}-\pi_{I}^{d e t}=\frac{\left(a^{2}+2 a b-b^{2}\right)^{2}}{8 b(b-a)}>0$. It also follows that, when $b \leq 2.4142 a$, entry will not be accommodated.

Proof of Proposition 3: Proposition 3(a) is immediate form the previous discussion. The proof of Proposition 3(b) is as follows.

For any given $c_{2}$, the entrant's profit can be expressed as

$$
\pi_{E}\left(c_{2}\right)=\int_{c_{1}\left(c_{2}\right)}^{c_{2}} c_{1}\left(c_{2}\right) z f(z) d z-K \text {. }
$$

As $c_{2} \rightarrow a, c_{1}\left(c_{2}\right) \rightarrow a$ and $\pi_{E}\left(c_{2}\right) \rightarrow-K<0$. As $c_{2} \rightarrow b, c_{1}\left(c_{2}\right) \rightarrow c_{1}^{* M}$ and $\pi_{E}\left(c_{2}\right) \rightarrow$ $\pi_{E}^{a c c}\left(c_{1}^{* M}, b\right)>0$, since $K<\hat{K}$. This follows from the fact that there exists a weak sequential equilibrium when entry occurs.

Now, $\pi_{E}\left(c_{2}\right)$ is continuous in $c_{2}$. Therefore, by the Intermediate Value Theorem, $\exists \hat{c}_{2} \in$ $(a, b)$ such that $\pi_{E}\left(\hat{c}_{2}\right)=0$. Further, since $\pi_{E}\left(c_{2}\right)$ is increasing in $c_{2}, \forall c_{2} \in\left[a, \hat{c}_{2}\right)$, $\pi_{E}\left(\hat{c}_{2}\right)<0$. That is, any $c_{2} \in\left[a, \hat{c}_{2}\right]$ deters entry. It is evident that, if $c_{2}>\hat{c}_{2}, \pi_{E}\left(\hat{c}_{2}\right)>0$ and, thus, entry will take place.

Proof of Corollary 1: First, let us consider that $0<a<b<\sqrt{3} a$ and $0 \leq K$. In this case, we have the following. $\bar{c}_{2}=a$, by (11). $\tilde{c_{1}}=a$, by $(6 \mathrm{a})$. Therefore, $\tilde{c_{2}}=c_{2}^{\prime}$, by (10). Further note that $c_{2}^{\prime} \geq a$, since $K \geq 0$ and $0<a<b$. Therefore, $c_{2}^{\text {det* }}=$ $\operatorname{Max}\left\{\operatorname{Min}\left\{\bar{c}_{2}, \tilde{c}_{2}\right\}, a\right\}=a$. Substituting $c_{2}=a$ in the expression of $\pi_{I}^{\text {det }}$, we get $\pi_{I}^{\text {det* }}=$ 
$\frac{a(a+b)}{2}$.

Next, let us consider that $0<\sqrt{3} a<b$. In this case, we have the following.

(i) If $0<K<\frac{a\left(b^{2}-3 a^{2}\right)}{6(b-a)}, a<c_{2}^{\prime}<\frac{b}{\sqrt{3}}$. Otherwise, if $\frac{a\left(b^{2}-3 a^{2}\right)}{6(b-a)} \leq K, c_{2}^{\prime} \geq \frac{b}{\sqrt{3}}$.

(ii) If $\frac{a^{3}}{27(b-a)}<K<\frac{b^{3}}{27(b-a)}, a<c_{2}^{\prime \prime}<\frac{b}{\sqrt{3}}$. Otherwise, if $\frac{b^{3}}{27(b-a)} \leq K, c_{2}^{\prime \prime} \geq \frac{b}{\sqrt{3}}$.

Therefore, when $0<\sqrt{3} a<b$ and $\tilde{c}_{1}=a, c_{2}^{\text {det* }}=\left\{\begin{array}{l}c_{2}^{\prime}, \text { if } \frac{a^{3}}{27(b-a)}<K<\frac{b^{3}}{27(b-a)} \\ \frac{b}{\sqrt{3}}, \text { if } \frac{a\left(b^{2}-3 a^{2}\right)}{6(b-a)} \leq K\end{array}\right.$, by (10), (11) and (12). However, when $0<\sqrt{3} a<b, \tilde{c}_{1}=a$ holds true provided that $0<a \leq c_{2} \leq \sqrt{3} a<b$ is satisfied. Now, $0<a \leq c_{2}^{\prime} \leq \sqrt{3} a<b$ and $0<K<\frac{a\left(b^{2}-3 a^{2}\right)}{6(b-a)}$ together implies that either $\left[0<\sqrt{3} a<b \leq 3 a\right.$ and $\left.0<K<\frac{a\left(b^{2}-3 a^{2}\right)}{6(b-a)}\right]$ or $[0<3 a<b$ and $\left.0<K \leq \frac{a^{3}}{(b-a)}\right]$. And, $0<a \leq \frac{b}{\sqrt{3}} \leq \sqrt{3} a<b$ and $0<K<\frac{a\left(b^{2}-3 a^{2}\right)}{6(b-a)}$ together implies that either $\left[0<\sqrt{3} a<b \leq 3 a\right.$ and $\left.K>\frac{a\left(b^{2}-3 a^{2}\right)}{6(b-a)}\right]$. Thus, we have the following.

- If $0<\sqrt{3} a<b \leq 3 a$ and $0<K<\frac{a\left(b^{2}-3 a^{2}\right)}{6(b-a)}, \tilde{c}_{1}=a$ and $c_{2}^{\text {det* }}=c_{2}^{\prime}$. Therefore $\pi_{I}^{\text {det* }}=\frac{\left(a^{2}+a b-2 K\right) \sqrt{a^{3}+2 K(b-a)}}{2 a^{3 / 2}}$.

- If $0<\sqrt{3} a<b \leq 3 a$ and $\frac{a\left(b^{2}-3 a^{2}\right)}{6(b-a)} \leq K<\hat{K}, \tilde{c}_{1}=a$ and $c_{2}^{\text {det* }}=\frac{b}{\sqrt{3}}$. Therefore $\pi_{I}^{\text {det* }}=\frac{b^{3}}{3 \sqrt{3}(b-a)}$.

- If $0<3 a<b$ and $0<K \leq \frac{a^{3}}{b-a}, \tilde{c}_{1}=a$ and $c_{2}^{\text {det* }}=c_{2}^{\prime}$. Therefore $\pi_{I}^{\text {det* }}=$ $\frac{\left(a^{2}+a b-2 K\right) \sqrt{a^{3}+2 K(b-a)}}{2 a^{3 / 2}}$.

It is also easy to check that when $0<\sqrt{3} a<b$ and $\tilde{c}_{1}=\frac{c_{2}}{\sqrt{3}}, c_{2}^{\text {det* }}=\left\{\begin{array}{l}c_{2}^{\prime \prime}, \text { if } \frac{a^{3}}{3 \sqrt{3}(b-a)}<K<\frac{b^{3}}{27(b-a)} \\ \frac{b}{\sqrt{3}}, \text { if } \frac{b^{3}}{27(b-a)} \leq K\end{array}\right.$, by (10), (11) and (12). But, when $0<\sqrt{3} a<b, \tilde{c}_{1}=\frac{c_{2}}{\sqrt{3}}$ holds true provided that $0<\sqrt{3} a<c_{2}<b$ is satisfied. Now, $0<\sqrt{3} a<c_{2}^{\prime \prime}<b$ and $\frac{a^{3}}{3 \sqrt{3}(b-a)}<K<\frac{b^{3}}{27(b-a)}$ together implies that $\left[0<3 a<b\right.$ and $\left.\frac{a^{3}}{(b-a)}<K<\frac{b^{3}}{27(b-a)}\right]$. And, $0<\sqrt{3} a<\frac{b}{\sqrt{3}}<b$ and $\frac{b^{3}}{27(b-a)} \leq K$ together implies that $\left[0<3 a<b\right.$ and $\left.\frac{b^{3}}{27(b-a)} \leq K\right]$. Thus, we have the following.

- If $0<3 a<b$ and $\frac{a^{3}}{(b-a)}<K<\frac{b^{3}}{27(b-a)}, \tilde{c}_{1}=\frac{c_{2}}{\sqrt{3}}$ and $c_{2}^{\text {det* }}=c_{2}^{\prime \prime}$. Therefore, 


$$
\pi_{I}^{\text {det* }}=\frac{\sqrt{3}}{2}\left\{\frac{b^{2} K^{1 / 3}}{(b-a)^{2 / 3}}-3 K\right\}
$$

- If $0<3 a<b$ and $\frac{b^{3}}{27(b-a)} \leq K<\hat{K}, \tilde{c}_{1}=\frac{c_{2}}{\sqrt{3}}$ and $c_{2}^{\text {det* }}=\frac{b}{\sqrt{3}}$. Therefore, $\pi_{I}^{\text {det } *}=$ $\frac{b^{3}}{3 \sqrt{3}(b-a)}$.

Proof of Proposition 4: It is straight forward to check that the solution of the incumbent's problem (13), ignoring the constraint (iii), is as follows.

$$
\begin{gathered}
c_{2}^{a c c}=\left\{\begin{array}{l}
\frac{\left(b^{2}-a^{2}\right)}{2 b}=c_{2}^{0}, \text { if } \tilde{c}_{1}=a \\
\frac{(\sqrt{13}-2) b}{3}=c_{2}^{00}, \text { if } \tilde{c}_{1}=\frac{c_{2}}{\sqrt{3}}
\end{array}\right. \\
\text { and } \pi_{I}^{a c c}=\left\{\begin{array}{l}
\frac{\left(a^{2}+b^{2}\right)^{2}}{8 b(b-a)}=\pi_{I}^{0}, \text { if } c_{2}^{a c c}=c_{2}^{0} \\
\frac{(13 \sqrt{13}-35) b^{3}}{81(b-a)}=\pi_{I}^{00}, \text { if } c_{2}^{a c c}=c_{2}^{00}
\end{array}\right.
\end{gathered}
$$

However, if $c_{2}=c_{2}^{0}, \tilde{c}_{1}=a$ holds provided that $\frac{c_{2}^{0}}{\sqrt{3}} \leq a<c_{2}^{0}<b$ holds true. This is because, corresponding to $c_{2}=c_{2}^{0}$, entrants most preferred choice is $c_{1}=\frac{c_{2}^{0}}{\sqrt{3}}$, if $a<\frac{c_{2}^{0}}{\sqrt{3}}<b$; otherwise, $c_{1}=a$. Now, $\frac{c_{2}^{0}}{\sqrt{3}} \leq a<c_{2}^{0}<b \Leftrightarrow 2.4142 a<b \leq 3.732 a$. Further, if $b<2.4142 a$, $c_{2}^{0}<a$ and, thus, entry will not be accommodated in this case.

If $c_{2}=c_{2}^{00}, \tilde{c}_{1}=\frac{c_{2}^{00}}{\sqrt{3}}$ holds provided that both $a<\frac{c_{2}^{00}}{\sqrt{3}}<b$ and $c_{2}^{00}<b$ are true, i.e. if $b>3.2364 a$ is true.

Now, if $3.2364 a<b<3.732 a$, we get (a) $\pi_{I}^{0}>\pi_{I}^{00}$, when $3.2364 a<b<3.474 a$ and (b) $\pi_{I}^{0}<\pi_{I}^{00}$, when $3.474 a<b<3.732 a$. Therefore, we have the following.

- $c_{2}=a$ and, thus, entry will not be accommodated, if $b<2.414 a$.

- $c_{2}=c_{2}^{0}, \tilde{c}_{1}=a$ and $\pi_{I}^{a c c}=\pi_{I}^{0}$, if $2.414 a<b<3.474 a$; provided that $c_{2}^{0}>c_{2}^{\prime}$, where $c_{2}^{\prime}$ is as in (7).

- $c_{2}=c_{2}^{00}, \tilde{c}_{1}=\frac{c_{2}}{\sqrt{3}}$ and $\pi_{I}^{a c c}=\pi_{I}^{00}$, if $3.474 a<b$; provided that $c_{2}^{00}>c_{2}^{\prime \prime}$, where $c_{2}^{\prime \prime}$ is as in (7). 
Sub-case (a): $0<a<b<\sqrt{3} a$

We have shown that, if $b<2.414 a$, it is optimal for the incumbent to choose $c_{2}=a$. Thus, if $0<a<b<\sqrt{3} a$, entry will not be accommodated in the equilibrium.

Sub-case (b): $0<\sqrt{3} a<b \leq 3 a$ and $0<K<\frac{a\left(b^{2}-3 a^{2}\right)}{6(b-a)}$

In this case $c_{2}=c_{2}^{\prime}$ deters entry.

It is now evident that, if $0<\sqrt{3} a<b<2.414 a, c_{2}^{0}<a$ and, thus, entry will not be accommodated. Clearly, if the variation in agents's types is small $(b<2.414 a)$, entry deterrence is optimal, regardless of the magnitude of the entrant's fixed cost.

When $2.414 a<b<3 a, c_{2}^{0}$ induces entry if $c_{2}^{0}>c_{2}^{\prime}$, which is possible if $0<K<$ $\frac{a b^{4}+a^{5}-6 a^{3} b^{2}}{8 b^{3}-8 a b^{2}}=K_{1}$, say. Further, $\pi_{I}^{\text {det }}<\pi_{I}^{a c c}$, if $0<K<K_{2}$; otherwise, if $K_{2}<K<K_{1}$, $\pi_{I}^{\text {det }}>\pi_{I}^{a c c}$

If $K>K_{1}, c_{2}^{0}<c_{2}^{\prime}$ and, thus, to accommodate entry the incumbent needs to set $c_{2}>c_{2}^{\prime}$. However, when $K>K_{1}, \pi_{I}^{d e t}>\pi_{I}^{a c c}$ and, thus, the incumbent's profit from setting $c_{2}>c_{2}^{\prime}$ to induce entry will be even lower than $\pi_{I}^{a c c}$. That is, if $K>K_{1}$, entry will not be accommodated.

Therefore, in this case, entry accommodation is preferred to entry deterrence when $2.414 a<$ $b<3 a$ and $0<K<K_{2}$.

Sub-case $(c): 0<\sqrt{3} a<b \leq 3 a$ and $\frac{a\left(b^{2}-3 a^{2}\right)}{6(b-a)} \leq K<\hat{K}$

Note that, in this case, under entry deterrence the incumbent sets $c_{2}=\frac{b}{\sqrt{3}}$.

Now, it is easy to observe that, if $2.414 a<b<3.474 a, c_{2}=c_{2}^{0}$ and $c_{1}=a$. But, $c_{2}^{0}<\frac{b}{\sqrt{3}}$.

So, to accommodate entry, $c_{2}$ must be greater that $\frac{b}{\sqrt{3}}$. However, we also have, $\pi_{I}^{\text {det }}>\pi_{I}^{a c c}$. Therefore, choosing $c_{2}$ greater than $c_{2}^{0}$ will lead to less profit for the incumbent than that in the case of entry deterrence. Thus, it is optimal for the incumbent to deter entry.

From sub-cases (a), (b) and (c), we can say that if the variation in agents' types is moderate $(2.414 a<b<3.474 a)$, then entry accommodation is optimal provided that the fixed 
cost of entry is less than a critical level $\left(0<K<K_{2}<K_{1}\right)$.

Sub-case (d): $0<3 a<b$ and $0<K \leq \frac{a^{3}}{b-a}$

In this case, the incumbent chooses $c_{2}=c_{2}{ }^{\prime}$ to deter entry. Now, if $3 a<b<3.474 a$, in the case of entry accommodation, ignoring the constraint (iii) of problem (13), we get $c_{2}=c_{2}^{0}, c_{1}=a$ and $\pi_{2}=\pi_{I}^{0}$. Upon inspection, we find that $c_{2}^{0}>c_{2}{ }^{\prime}$, if $0<K<K_{1}$. And, $\pi_{I}^{a c c}>\pi_{I}^{\text {det }}$, if $0<K<K_{2}<K_{1}$. So, if $3 a<b<3.474 a$ and $0<K<K_{2}$, entry accommodation is optimal for the incumbent.

Next, consider that $0<3.474 a<b$. In the case of entry accommodation, ignoring the constraint (iii) of problem (13), we get $c_{2}=c_{2}^{00}$ and $c_{1}=\frac{c_{2}}{\sqrt{3}}$. Corresponding profit of the incumbent is $\pi_{I}^{a c c}=\pi_{I}^{00}$. It follows that $c_{2}^{00}>c_{2}{ }^{\prime}$ holds for all $K$ such that $0<K \leq \frac{a^{3}}{b-a}$. And, $\pi_{I}^{a c c}>\pi_{I}^{d e t}$, if $K<\operatorname{Min}\left\{K_{3}, \frac{a^{3}}{b-a}\right\}$; where $K_{3}\left(>K_{2}\right)$ is given by the first root of the following equation.

$-6561 a^{9}+13122 a^{7} b^{2}-6561 a^{5} b^{4}+13688 a^{3} b^{6}-3640 \sqrt{13} a^{3} b^{6}+\left(39366 a^{7}-39366 a^{6} b-\right.$ $\left.52488 a^{5} b^{2}+52488 a^{4} b^{3}+13122 a^{3} b^{4}-13122 a^{2} b^{5}\right) K+\left(-78732 a^{5}+157464 a^{4} b-26244 a^{3} b^{2}-\right.$ $\left.104976 a^{2} b^{3}+52488 a b^{4}\right) K^{2}+\left(52488 a^{3}-157464 a^{2} b+157464 a b^{2}-52488 b^{3}\right) K^{3}=0$.

So, when $0<3.474 a<b$ and $0<K=\frac{a^{3}}{b-a}$, entry accommodation is optimal unless the fixed cost of entry is greater than a critical value.

Sub-case $(e): 0<3 a<b$ and $\frac{a^{3}}{(b-a)}<K<\frac{b^{3}}{27(b-a)}$

In the case of entry deterrence, we have $c_{2}=c_{2}{ }^{\prime \prime}$ and $c_{1}=\frac{c_{2}}{\sqrt{3}}$.

If $3 a<b<3.474 a$, in the case of entry accommodation, ignoring the constraint (iii) of problem (13), we get $c_{2}=c_{2}^{0}, c_{1}=a$ and $\pi_{2}=\pi_{I}^{0}$. But, $c_{2}^{0}<c_{2}^{\prime \prime}$ and $\pi_{I}^{a c c}<\pi_{I}^{\text {det }}$. Clearly, entry deterrence is optimal.

Next, consider that $0<3.474 a<b$. In the case of entry accommodation, ignoring the constraint (iii) of problem (13), we get $c_{2}=c_{2}^{00}$ and $c_{1}=\frac{c_{2}}{\sqrt{3}}$. Corresponding profit of the 
incumbent is $\pi_{I}^{a c c}=\pi_{I}^{00}$. Now, $c_{2}^{00}-c_{2}^{\prime \prime}>0$, if $\frac{b^{3}}{27(b-a)}<K<\frac{b^{3}(25 \sqrt{13}-86)}{81 \sqrt{3}(b-a)}=K_{4}<\frac{b^{3}}{27(b-a)}$. And, when $c_{2}^{00}-c_{2}{ }^{\prime \prime}>0$, we have $\pi_{I}^{a c c}>\pi_{I}^{\text {det }}$, if $0<3.474 a<b$ and $\frac{a^{3}}{27(b-a)}<K<K_{5}$; where $K_{5}<K_{4}$. But, when $c_{2}^{00}-c_{2}^{\prime \prime}<0, \pi_{I}^{a c c}<\pi_{I}^{\text {det }}$. Thus, entry accommodation is optimal, if $0<3.474 a<b$ and $\frac{a^{3}}{27(b-a)}<K<K_{5}$.

Sub-case $(f): 0<3 a<b$ and $\frac{b^{3}}{27(b-a)} \leq K<\hat{K}$

In the case of entry deterrence, $c_{2}=\frac{b}{\sqrt{3}}$ and $c_{1}=\frac{c_{2}}{\sqrt{3}}$.

If $3 a<b<3.474 a$, in the case of entry accommodation, ignoring the constraint (iii) of problem (13), we get $c_{2}=c_{2}^{0}, c_{1}=a$. But, $c_{2}^{0}<\frac{b}{\sqrt{3}}$ and $\pi_{I}^{a c c}<\pi_{I}^{\text {det }}$. Clearly, entry deterrence is optimal.

Next, consider that $0<3.474 a<b$. In the case of entry accommodation, ignoring the constraint (iii) of problem (13), we get $c_{2}=c_{2}^{00}$ and $c_{1}=\frac{c_{2}}{\sqrt{3}}$. Further, it is easy to check that $c_{2}^{00}<\frac{b}{\sqrt{3}}$ and $\pi_{I}^{a c c}<\pi_{I}^{d e t}$. Thus, entry deterrence is optimal.

From the results obtained in sub-case (a) - (f), Proposition 4 is immediate. 


\section{References}

1. Ahuvia, A. C., Adelman, M. P., 1992. Formal Intermediaries in the Marriage Market: A Typology and Review. Journal of Marriage and Family 54, 452-463.

2. Agrawal, A., 2015. Cyber-matchmaking among Indians: Re-arranging marriage and doing 'kin work'. South Asian Popular Culture 13(1), 15-30.

3. Aghion, P., Bolton, P., 1987. Contracts as a barrier to entry. American Economic Review 77(3), 388-401.

4. Armstrong, M., 2006. Competition in Two-sided Markets. Rand Journal of Economics 37(3), 668-691.

5. Baumol, W., J. Panzar and R. Willig. 1982. Contestable Markets and the Theory of Industry Structure. New York: Harcourt Brace Jovanovich.

6. Becker, G., 1973. A Theory of Marriage: Part I. Journal of Political Economy 81, 813-846.

7. Caillaud, B., Jullien, B., 2001(a). Competing Cybermediaries. European Economic Review (Papers and Proceedings) 45(4-6), 797-808.

8. Caillaud, B., Jullien, B., 2003. Chicken \& Egg: Competition among Intermediation Service Providers. The Rand Journal of Economics 34(2), 309-328.

9. Dixit, A., 1979. A Model of Duopoly Suggesting a Theory of Entry Barriers. Bell Journal of Economics 10(1), 20-32.

10. Dixit, A., 1980. The Role of Investment in Entry-Deterrence. The Economic Journal 90(357), 95-106.

11. Fudenberg, D., Tirole., 1984. The Fat-Cat Effect, the Puppy-Dog Ploy, and the Lean and Hungry Look. The American Economic Review 74(2), Papers and Proceedings of the Ninety-Sixth Annual Meeting of the American Economic Association, 361-366. 
12. Gale, D., Shapley, L S., 1962. College admissions and the stability of marriage. American Mathematical Monthly 69(1), 9-15.

13. Lee, R. S., 2014. Competing Platforms. Journal of Economics and Management Strategy 23(3), 507-526.

14. Lee, R. S., 2013. Vertical Integration and Exclusivity in Platform and Two-sided Markets. American Economic Review 103(7), 2960-3000.

15. Milgrom, P., Roberts, J., 1982. Limit Pricing and Entry Under Incomplete Information: An Equilibrium Analysis. Econometrica 50 (2), 443-459

16. Pal, R., Saha, B., 2006. Wage Commitment, Signalling, and Entry Deterrence or Accommodation. LABOUR 20(4), 625-650.

17. Pal, R., Saha, B., 2008. Union-oligopoly bargaining and entry deterrence: a reassessment of limit pricing. Journal of Economics 95(2), 121-147.

18. Ramani, V., 2015. Existence of Multiple Matchmakers in a Two-sided Matching Market. Studies in Microeconomics (forthcoming).

19. Roth, A., Sotomayor, M., 1990. Two-Sided Matching: A Study in Game- Theoretic Modelling and Analysis. Econometric Society Monograph No. 18, Cambridge; New York and Melbourne, Cambridge University Press.

20. Shako, S., 2004. New Cultural Structures: South Asian Matrimonial Websites. McMaster Journal of Communication 1 (1), 57-63.

21. Spence, A.M., 1977. Entry, Capacity, Investment, and Oligopolistic Pricing. Bell Journal of Economics 8(2), 534-544.

22. Spence, A.M., 1979. Investment Strategy and Growth in a New Market. Bell Journal of Economics 10(1), 1-19.

23. Titzamann, F., 2013. Changing Patterns of Matchmaking: The Indian Online Matrimonial Market. Asian Center for Womens Studies 19(4), 64-94. 